\title{
Rate Multiplication and Two-group Resource Allocation in Multi-code CDMA Networks
}

\author{
Hadhrami Ab. Ghani \\ Department of Electrical and \\ Electronic Engineering \\ Imperial College London \\ South Kensington Campus, London SW7 2AZ \\ Email: hadh.ghani05@imperial.ac.uk
}

\author{
M. K. Gurcan \\ Department of Electrical and \\ Electronic Engineering \\ Imperial College London \\ South Kensington Campus, London SW7 2AZ \\ Email: m.gurcan@imperial.ac.uk
}

\begin{abstract}
The residual energy in multi-code CDMA systems is not used to transmit any useful information and may increase up to the incremental energy multiplied with the number of multi-code channels. A two-group resource allocation scheme is developed to reduce this upper bound on the residual energy down to as low as a single-channel incremental energy, resulting higher energy utilization to improve the sum capacity. Since the incremental energy is the additional energy required to increase a data rate to a higher data rate by an additional amount called bit granularity, a new design of modulation and coding scheme based on the rate multiplication of outer and inner codes can be developed to produce different data rates such that the bit granularity is reduced, hence decreasing the incremental energy to further maximize the sum capacity. The two-group resource allocation scheme implemented with the proposed modulation and coding scheme is observed to have a $4 \mathrm{~dB}$ gain over the current HSDPA systems.
\end{abstract}

\section{INTRODUCTION}

The rapid growth in the number of mobile radio users [1] arouses much research interest in the development of mobile radio systems. Demands for systems supporting various multirate services such as multimedia applications render the incorporation of multi-code transmission in code division multiple access (CDMA) networks [2]. One of the leading mobile radio technologies which operates on multi-code CDMA networks is the high speed downlink packet access (HSDPA) system. With limited radio resources available, a novel transmission model is presented in this paper aiming to improve the sum capacity by capitalizing on the residual energy which remains unused in the current HSDPA systems.

The residual energy, defined as the amount of energy not used to transmit any useful information, is upper-bounded by the number of employed multi-code channels multiplied with the incremental energy, which is the additional energy required to increase a given data rate by an amount of incremental data rate called bit granularity. Given $K$-multi-code white Gaussian CDMA channels, a total signal energy, a noise variance, a channel response and a capacity gap [3], a two-group resource allocation scheme implemented with a novel modulation and coding scheme (MCS) design is presented in this paper to improve the sum capacity by lowering the upper bound on the residual energy down to a single-channel incremental energy, as opposed to the incremental energy multiplied with the number of multi-code channels.

In literature, various strategies are proposed to utilize the available radio resources such as in [4]-[6], to name a few. In [7], a two-best user scheduling technique is designed according to a proportional fairness index [8], given a limited number of multi-codes and MCSs. This approach is different than the approach presented in this paper since only one type of MCSs is selected. A few scheduling techniques are proposed to maximize the transmission data rate by considering the number of multi-codes, the types of MCSs, [9], the transmit power [10] and maximum allowable bit rates [11]. Although various constraints are considered, the issue of residual energy reduction has not been addressed. The two-group resource allocation scheme has been successfully employed in [12] to improve the throughput in ad-hoc networks and in [13], [14] to maximize the throughput in overloaded CDMA systems. On the other hand, the two-group resource allocation scheme presented in this paper attempts to reduce the upper bound on the residual energy to a single-channel incremental energy, hence maximizing the sum capacity.

Since the incremental energy is a function of the bit granularity, it can be further reduced by decreasing this parameter value. HSDPA systems, as stated in the Third Generation Partnership Project (3GPP) Release 5 [15], employ quadrature phase shift keying (QPSK) and 16-QAM (quadrature amplitude modulation) schemes incorporated with Turbo codes of rates $1 / 4,1 / 2$ and $3 / 4$ [16], [17]. The effective data rates of these MCSs yield bit granularities of 0.5 and 1 bits per channel. In this paper, a novel MCS design aiming to realize bit granularities of 0.25 bits per channel is proposed which incorporate coded parity packet (CPP) codes [18] with Turbo codes and $M$-QAM schemes, where $M=4,16,64$, and 256 .

The rest of this paper is organized as follows. Section II gives an account for the fundamental concepts of the residual and the incremental energy, highlighting the necessary improvement proposed to address the residual energy utilization problem. In Section III the two-group resource allocation scheme is presented to solve this problem before being further enhanced with a new MCS design in Section IV which reduces the bit granularity. Section V presents the numerical results 
and Section VI concludes this paper.

\section{The Residual EnERgy UpPer Bound}

In $K$-multi-code CDMA systems such as HSDPA, the residual energy is upper-bounded by the incremental energy multiplied with the number of multi-code channels, as will be shown here. With $K$ white Gaussian multi-code channels, a total signal energy of $\varepsilon_{T}$ and a set of $N_{P}$ MCSs which operate at $b_{p}, p=1,2, \ldots, N_{P}$ bits per channel, the incremental energy, $e_{p}\left(b_{p}\right)$ can be calculated as

$$
\begin{aligned}
e_{p}\left(b_{p}\right) & =\varepsilon_{p+1}\left(b_{p}+\beta_{p}\right)-\varepsilon_{p} b_{p} \\
& =\frac{2 \Gamma_{p} \sigma^{2} 2^{b_{p}+1}\left(2^{\beta_{p}}-1\right)}{|h|^{2}}
\end{aligned}
$$

with

$$
\varepsilon_{p}\left(b_{p}\right)=\frac{2 \Gamma_{p} \sigma^{2}\left(2^{b_{p}}-1\right)}{|h|^{2}},
$$

as the energy required by $\mathrm{MCS}_{p}$ to realize $b_{p}$ such that

$$
\begin{aligned}
K \varepsilon_{p}\left(b_{p}\right) & \leq \varepsilon_{T}<K \varepsilon_{p+1}\left(b_{p}+\beta_{p}\right), \\
\varepsilon_{1}\left(b_{1}\right) & <\varepsilon_{2}\left(b_{2}\right)<\cdots<\varepsilon_{N_{P}}\left(b_{N_{P}}\right), \\
b_{1} & <b_{2}<\cdots<b_{N_{P}}
\end{aligned}
$$

where $h$ is the channel response, $\beta_{p}=b_{p+1}-b_{p}$ is the bit granularity, $\sigma^{2}$ is the noise variance and $\Gamma_{p}=\frac{\operatorname{SNR}_{p}}{2^{b_{p}}-1}, \Gamma_{p} \approx$ $\Gamma_{p+1}=\Gamma, \forall p \in\left\{1,2, \ldots, N_{P}-1\right\}$ is the capacity gap [3]. $\mathrm{SNR}_{p}$ is the signal-to-noise ratio (SNR) required by $\mathrm{MCS}_{p}$ to realize $b_{p}$ at a bit error rate of $1 \times 10^{-5}$. Using (2) and (3), the residual energy, $e_{p}^{R}$ can be written as

$$
0 \leq e_{p}^{R}=\varepsilon_{T}-K \varepsilon_{p}\left(b_{p}\right)<K e_{p}\left(b_{p}\right),
$$

which proves the upper-bound on the residual energy in HSDPA systems claimed in the beginning of this section.

Given $K, h, \sigma^{2}, \Gamma_{p}$ and $\varepsilon_{T}$, the allocated data rate, $b_{p}$ can be calculated using (2) and (3) to yield the sum capacity, $C_{T}$ and the total $\mathrm{SNR}, \mathrm{SNR}_{T}$ in the current HSDPA systems as

$$
\begin{aligned}
C_{T} & =K b_{p}, \\
\mathrm{SNR}_{T} & =K \Gamma_{p}\left(2^{b_{p}}-1\right),
\end{aligned}
$$

respectively. Since the sum capacity, $C_{T}$ is taken as the performance parameter, a $C_{T}$ vs. $\mathrm{SNR}_{T}$ graph based on (7) and (8) is plotted in Section $\mathrm{V}$ along with the improved sum capacity obtained using the two-group resource allocation approach, as will be discussed in the following section.

\section{THE RESIDUAL ENERGY REDUCTION}

The multiplicative factor of $K$ multi-code channels lead to a significant increase in the residual energy in HSDPA systems, as shown in (6). For this reason, a two-group resource allocation scheme is proposed in this section to reduce the upper bound on the residual energy down to only a single-channel incremental energy, given approximately equal capacity gaps, $\Gamma_{p} \approx \Gamma_{p+1}$

The proposed two-group resource allocation scheme is shown in Figure 1. Once the estimated SNR information is received from the receiver end, all $K$ channels are divided into two groups, where $\mathrm{MCS}_{p}$ with a data rate of $b_{p}$ each is chosen for each channel in the first group of $K-L$ channels and $\mathrm{MCS}_{p+1}$ with a data rate of $b_{p+1}$ each is chosen for each channel in the second group of $L$ channels. By combining the two groups, an improved amount of energy,

$$
\begin{aligned}
\varepsilon_{p}^{C_{T}} & =(K-L) \varepsilon_{p}\left(b_{p}\right)+L \varepsilon_{p+1}\left(b_{p+1}\right), \\
& =K \varepsilon_{p}\left(b_{p}\right)+L e_{p}\left(b_{p}\right),
\end{aligned}
$$

is utilized to transmit data, where the number of channels in the second group, $L$ satisfies (13), and $\Gamma_{p} \approx \Gamma_{p+1}=\Gamma, \forall p \in$ $\left\{1,2, \ldots, N_{P}-1\right\}$. An additional amount of energy, $L e_{p}\left(b_{p}\right)$ is utilized as compared to the current HSDPA systems, rendering a new reduced residual energy, $e_{p}^{R^{\prime}}=\varepsilon_{T}-K \varepsilon_{p}\left(b_{p}\right)-L e_{p}\left(b_{p}\right)$.

The input data which have been allocated by this scheme are then coded, modulated and spread before being transmitted. An important observation on (13) is the reduction in the upper bound energy down to only a single-channel incremental energy, $e_{p}\left(b_{p}\right)$, as opposed to the incremental energy multiplied with the number of channels, $K e_{p}\left(b_{p}\right)$, as can be seen in (6), hence proving the claim made earlier on.

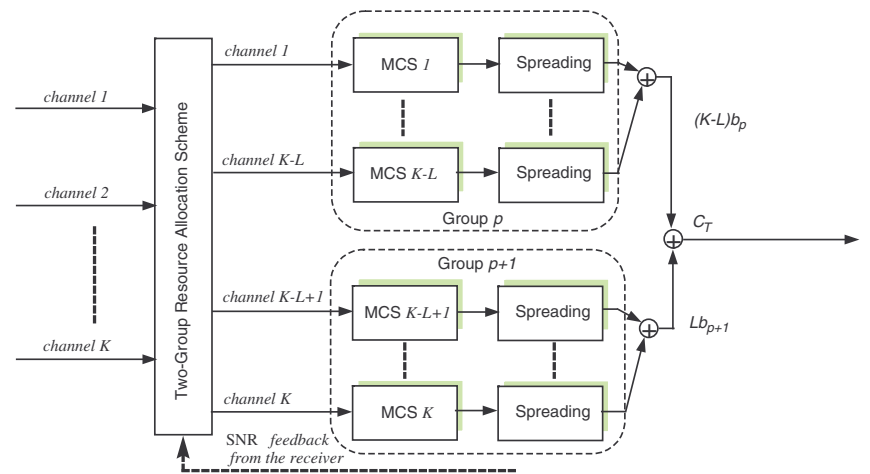

Fig. 1. An illustration of the two-group resource allocation scheme implementation

To measure system performance, the resultant sum capacity, $C_{T}^{\prime}$ for this system is plotted over a range of SNRs, $\mathrm{SNR}_{T}^{\prime}$

$$
\begin{aligned}
(K-L) \varepsilon_{p}\left(b_{p}\right)+L \varepsilon_{p+1}\left(b_{p+1}\right) & \leq \varepsilon_{T}<(K-L-1) \varepsilon_{p}\left(b_{p}\right)+(L+1) \varepsilon_{p+1}\left(b_{p+1}\right), \\
\varepsilon_{p}^{C_{T}} & \leq \varepsilon_{T}<\varepsilon_{p}^{C_{T}}+e_{p}\left(b_{p}\right), \\
0 & \leq \varepsilon_{T}-K \varepsilon_{p}\left(b_{p}\right)-L e_{p}\left(b_{p}\right)<e_{p}\left(b_{p}\right),
\end{aligned}
$$


in Section V. Given $K, h, \sigma^{2}, \Gamma_{p}, \varepsilon_{T}$, and $b_{p}$, which can be calculated using (2) and (3), the number of channels in the second group, $L$ can be computed using (13) to yield $C_{T}^{\prime}$ and $\mathrm{SNR}_{T}^{\prime}$ as

$$
\begin{gathered}
C_{T}^{\prime}=(K-L) b_{p}+L b_{p+1}, \\
\operatorname{SNR}_{T}=(K-L) \Gamma_{p}\left(2^{b_{p}}-1\right)+L \Gamma_{p}\left(2^{b_{p+1}}-1\right),
\end{gathered}
$$

respectively. Hence, the new sum capacity, $C_{T}^{\prime}$, is $L \beta_{p}$ bits per channel larger than the original sum capacity in HSDPA systems, $C_{T}$ as shown in (7).

Since the incremental energy is also a function of the bit granularity, $\beta_{p}$, as given in (1), a further reduction in the residual energy, can be achieved if this parameter value is reduced. In Section IV, a new design of MCSs is proposed to reduce the bit granularity to as low as 0.25 bits per channel, as compared to the current minimum bit granularity of 0.5 bits per channel in HSDPA systems [16].

\section{INCREMENTAL ENERGY REDUCTION}

In this section, a new MCS design is put forward to reduce the incremental energy by decreasing the bit granularity while maintaining the capacity gap value within $2 \mathrm{~dB}$ in order to maximize the sum capacity. This is realized by incorporating an $M$-QAM modulation scheme with a Turbo encoder of rate $r_{I, \alpha}, \alpha=1,2, \ldots N_{I}$ and a CPP encoder of rate $r_{O, V}, V \in \mathbb{R}$ such that the effective data rate, $b_{p}$ becomes

$$
b_{p}=r_{O, \kappa} r_{I, \alpha} \log _{2} M .
$$

As widely proposed in literature, the standard Turbo code rates employed here can be taken as, $r_{I, \alpha},=\alpha /(\alpha+1), \alpha=$ $1,2,3$. With these Turbo code rates, a set of CPP codes are designed in order to achieve bit granularities as low as 0.25 bits per channel. To check the integrity of the received frames, the source frames are first cyclic redundancy check (CRC) encoded. If $V$ source frames are encoded, the corresponding CPP code rate that should be used to produce the target bit granularity of 0.25 bits per channel is $r_{O, V}=V /(V+1), V=$ $2,3,4,5,7,11$. The CPP encoding process combines the $V$ source frames using bit-wise XOR combinations to produce a parity frame. Although XOR combination is used, this approach is different from [19] since a fixed XOR combination of source frames is used, hence a unique code rate is achieved. A set of effective code rates of this proposed MCS design with a minimum bit granularity, $\beta_{\min }=0.25$, and capacity gap values of less than $2 \mathrm{~dB}$ are recorded in Table I.

After the CPP encoding process is performed, the encoded frames are Turbo encoded. The Turbo encoded vectors produced are $\left(N_{I}+K_{C}\right)$-bit vectors, $\underline{x}_{e}^{(c)}=$ $\left[x_{e, 1}^{(c)}, x_{e, 2}^{(c)} \ldots, x_{e, N_{I f}+K_{C}}^{(c)}\right], e=1,2, \ldots, E$, which are formed by $N$-bit systematic vectors, $\underline{x}_{e}^{(u)}=\underline{u}_{e}$ and two $\left(\frac{N_{I f}+K_{C}-N}{2}\right)$-bit parity vectors, $\underline{x}_{e}^{(p 1)}$ and $\underline{x}_{e}^{(p 2)}$, where $E=$ $V+1$ is the total number of parity packets produced and $N_{I f}$ is the original information frame length.

A brief account for the iterative decoding scheme employed for Turbo-CPP codes is given in the next sub-section.

\section{A. The Iterative Decoding Scheme}

With an outer code rate of $V /(V+1)$, the iterative decoding scheme employs one multiple frame detector, in which $V+$ 1 Turbo decoders iteratively interact between each other to decode $V$ source frames. If $V=2$, there are three Turbo decoders required, which are referred with a subscript $e$, where $e \in\{1,2,3\}$, as shown in Figure 2. As can be seen in this figure, two different soft bits are fed to each Turbo decoder, which can be calculated using the formula given in [17]. The first type represents the channel soft information, $\Lambda\left(u_{e, n}\right)$ of the systematic bit, $u_{e, n}$ and the second type corresponds to the channel soft informations, $\Lambda\left(p_{e, n}^{(1)}\right)$ and $\Lambda\left(p_{e, n}^{(2)}\right)$, for both the first and and the second parity bits, $p_{e, n}^{(1)}$ and $p_{e, n}^{(2)}$.

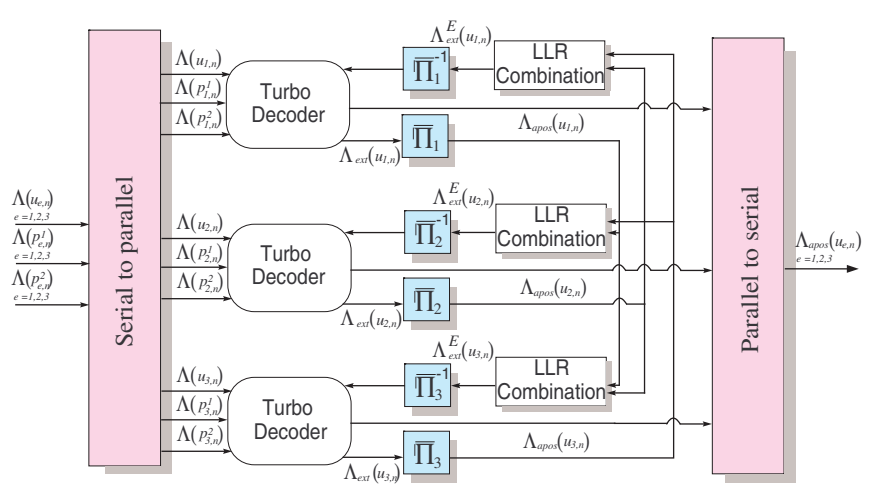

Fig. 2. A multiple frame detector with $V=2$ and $E=V+1=3$

Every Turbo decoder employs two component decoders, which both run a modified log-MAP algorithm [20]. After every internal decoding iteration inside a Turbo decoder, a soft information called extrinsic log-likelihood ratio (LLR), $\Lambda_{e x t}\left(u_{e, n}\right)$ is produced as

$$
\Lambda_{\text {ext }}\left(u_{e, n}\right)=\Lambda_{\text {ext }}\left(u_{e, n}^{(2)}\right) \triangleq \Lambda_{\text {apos }}\left(u_{e, n}^{(2)}\right)-\Lambda_{\text {priori }}\left(u_{e, n}^{(2)}\right) .
$$

This LLR value is generated by the second component decoder, denoted with the superscript (2), before being passed to the other Turbo decoders in the multiple frame detector as can be seen in Figure 2. The extrinsic LLRs received by every Turbo decoder from the rest of the Turbo decoders are combined as an external extrinsic LLR, $\Lambda_{e x t}^{E}\left(u_{e, n}\right)$. If Turbo decoder for source frame 1 is taken as an example with $V=2$ source frames, the resultant external extrinsic LLR, $\Lambda_{\text {ext }}^{E}\left(u_{1, n}\right)$, received by this Turbo decoder can be written as,

$\Lambda_{\text {ext }}^{E}\left(u_{1, n}\right)=2 \arctan \left(\tanh \left(\Lambda_{\text {ext }}\left(u_{2, n}\right)\right) \tanh \left(\Lambda_{\text {ext }}\left(u_{3, n}\right)\right)\right)$,

which becomes a new source of a priori information in the next iteration. Hence, the updated a priori informations for both component decoders in every new iteration are given as

$$
\begin{aligned}
& \Lambda_{\text {apriori }}\left(u_{1, n}^{(1)}\right)=\Lambda_{\text {ext }}\left(u_{1, n}^{(2)}\right)+\Lambda\left(u_{1, n}\right)+\Lambda_{\text {ext }}^{E}\left(u_{1, n}\right) \\
& \Lambda_{\text {apriori }}\left(u_{1, n}^{(2)}\right)=\Lambda_{\text {ext }}\left(u_{1, n}^{(1)}\right)+\Lambda\left(u_{1, n}\right)+\Lambda_{\text {ext }}^{E}\left(u_{1, n}\right)
\end{aligned}
$$

After a pre-determined number of iterations, the main soft output of each Turbo decoder known as the a posteriori LLR, 
$\Lambda_{\text {apos }}\left(u_{e, n}^{(2)}\right)$ is produced as

$\Lambda_{\text {apos }}\left(u_{e, n}^{(2)}\right)=\log \left(\frac{\left(u_{e, n}=+1 \mid \Lambda\left(p_{e, n}^{(2)}\right), \Lambda_{\text {priori }}\left(u_{e, n}^{(2)}\right)\right)}{\left(u_{e, n}=-1 \mid \Lambda\left(p_{e, n}^{(2)}\right), \Lambda_{\text {priori }}\left(u_{e, n}^{(2)}\right)\right)}\right)$,

The CRC algorithm can be then invoked on the sign of $\Lambda_{\text {apos }}\left(u_{e, n}^{(2)}\right)$. If one out of $V+1$ frames is still in error, a hard decoding can be applied by performing a bit-wise XOR combination on the remaining $V$ successfully decoded frames. These Turbo-CPP QAM schemes are configured to realize a minimum bit granularity, $\beta_{\min }=0.25$ (see Table II) with capacity gap values of less than $2 \mathrm{~dB}$ before being implemented with the two-group resource allocation scheme in Section V-A to observe the improved sum capacities over a range of SNRs. In the following section, relevant numerical results pertaining to the proposed schemes are presented.

\section{NuMERicAl RESUlts}

Before simulating the two-group resource allocation scheme, a comparison study is run to demonstrate the advantage of using the proposed MCS over different MCS designs in literature in reducing bit granularities while maintaining low capacity gap values to reduce the residual energy. With QAM schemes, these MCSs employ the standard Turbo codes, Multiple Turbo Fountain codes [21] and BCH-Turbo codes.

In Table I, combinations of outer code rates, $r_{O}$, inner code rates $r_{I}$, and constellation sizes, $M$ are given to achieve the data rates, $b_{p}$ or spectral efficiencies, $R / W$ in Figure 3. For MCSs employing a single type of channel code, the code rate is represented as $r_{c}$. The acronym MTF QAM represents an MCS design which employs Multiple Turbo Fountain codes. $\mathrm{BCH}(127,57)$ and $\mathrm{BCH}(255,187)$ codes are concatenated to generate $r_{O}=\frac{2}{3}$ in BCH-Turbo QAM schemes.

With 3072-bit length source frames, a scatter diagram, $\frac{E_{b}}{N_{0}}$ vs. $\frac{R}{W}$ for each MCS is plotted in Figure 3, where $\frac{E_{b}}{N_{0}}=\frac{1}{b_{p}} \mathrm{SNR}_{p}$ is the recorded bit energy-to-noise ratio when a bit error rate of $1 \times 10^{-5}$ is hit and $\frac{R}{W}=\eta=b_{p}$ is the spectral efficiency. The lower bound curve in this figure constitutes the Shannon's capacity bound, where $\frac{E_{b}}{N_{0}}=\frac{2^{\eta}-1}{\eta}$. It can be seen that the MCSs which employ BCH-Turbo codes exhibit the worst performance with the largest capacity gap. The MCSs which employ Multiple Turbo Fountain codes produce smaller capacity gap values but the corresponding rate adjustment to generate multiple code rates to further reduce the bit granularities with only one type of channel codes used is computationally inefficient. The same problem in rate adjustment also suffered in Turbo-QAM schemes, although the corresponding capacity gap values are lower. The proposed Turbo-CPP QAM schemes are observed to produce capacity gap values as low as those of the Turbo-QAM schemes, but with an added advantage of simplified rate adjustment since multiple effective code rates can be realized by multiplying the inner and outer code rates. Hence, these proposed MCSs can be used in the two-group resource allocation scheme to further reduce the residual energy.

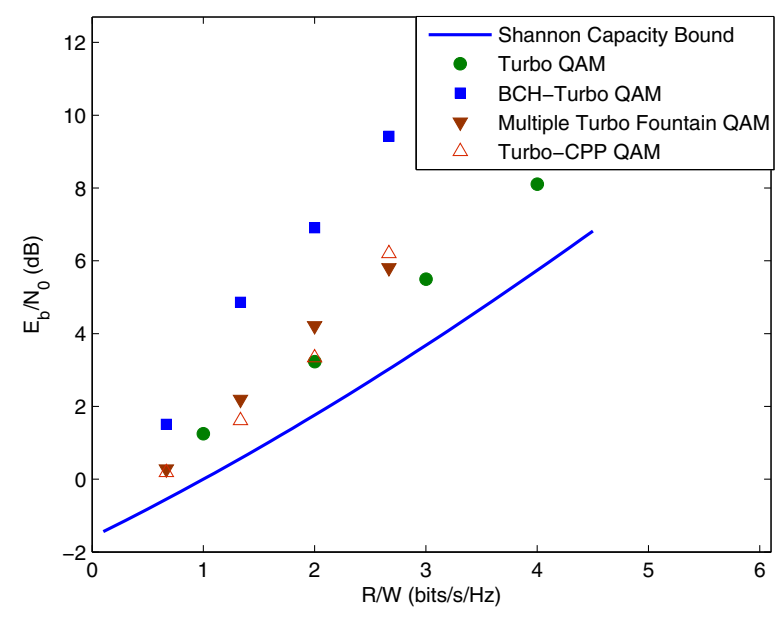

Fig. 3. The required $\frac{E_{b}}{N_{0}}$ to achieve bit error rates of $1 \times 10^{-5}$ at different spectral efficiencies, $R / W$ (bits/s/Hz) for different MCSs at a frame size of 3072 bits

\begin{tabular}{l|c|c|c|c|c|c|c|c|c|c}
\hline \hline \multirow{2}{*}{$M$} & \multicolumn{2}{|c|}{ BCH-Turbo QAM } & \multicolumn{2}{c|}{ MTF QAM } & \multicolumn{2}{|c|}{ Turbo-CPP QAM } & \multicolumn{2}{|c|}{ Turbo QAM } \\
\cline { 2 - 10 } & $r_{O}$ & $r_{I}$ & $b_{p}$ & $r_{c}$ & $b_{p}$ & $r_{O}$ & $r_{I}$ & $b_{p}$ & $r_{c}$ & $b_{p}$ \\
\hline \hline 4 & $2 / 3$ & $1 / 2$ & 0.67 & $1 / 3$ & 0.67 & $2 / 3$ & $1 / 2$ & 0.67 & $1 / 2$ & 1.00 \\
16 & $2 / 3$ & $1 / 2$ & 1.33 & $1 / 3$ & 1.33 & $2 / 3$ & $1 / 2$ & 1.33 & $1 / 2$ & 2.00 \\
64 & $2 / 3$ & $1 / 2$ & 2.00 & $1 / 3$ & 2.00 & $2 / 3$ & $1 / 2$ & 2.00 & $1 / 2$ & 3.00 \\
256 & $2 / 3$ & $1 / 2$ & 2.67 & $1 / 3$ & 2.67 & $2 / 3$ & $1 / 2$ & 2.67 & $1 / 2$ & 4.00 \\
\hline \hline
\end{tabular}

TABLE I

THE MODULATION AND CODING SCHEMES SPECIFICATIONS

\section{A. Improvement in The Sum Capacity}

The two-group resource allocation scheme is simulated with the proposed MCSs to demonstrate the increase in the sum capacity when the residual energy is reduced. A $K$ multi-code CDMA system is considered with $K=15$ and a speading factor, $S_{F}=16$. Rate multiplication for these MCSs as discussed in Section V is implemented to realize the minimum bit granularity, $\beta_{\min }=0.25$ bits per channel. Five data rates of MCSs [16] used in HSDPA systems (HSDPA MCSs) with $\beta_{\min }=0.5$ bits per channel are also tested for comparison. Using the two-group resource allocation scheme, the sum capacity can be obtained using (14) and the total SNR, $\mathrm{SNR}_{T}$ is obtained using (15). For HSDPA MCSs, the sum capacity and the total $\mathrm{SNR}_{T}$ are calculated using (7) and (8) respectively.

Table II shows the data rates realized in both collections of MCSs, along with the corresponding combinations of outer code rates, $r_{O, V}$, inner code rates, $r_{I, \alpha}$ and the constellation sizes, $M$ for the proposed Turbo-CPP QAM scheme. Two data rates, $b_{p}=3.20,3.50$ are not selected since the corresponding capacity values are greater than $2 \mathrm{~dB}$. The sum capacities, $C_{T}$ and the required total SNRs, $\mathrm{SNR}_{T}$ for both Turbo-CPP QAM schemes and HSDPA MCSs are plotted in Figure 4. In bits per second, the sum capacity can be calculated as, $C_{T b p s}=$ $\frac{W}{S_{F}} C_{T}$, where $W=3.84 \mathrm{Mchips} / \mathrm{s}$ is the chip rate.

Without the two-group resource allocation scheme, the pro- 


\begin{tabular}{c|c|c|c|c||c|c|c|c|c}
\hline \hline \multicolumn{1}{c}{ HSDPA MCS } & \multicolumn{6}{c}{ Turbo-CPP } \\
\hline$r_{O, \kappa}$ & $r_{I, \alpha}$ & $M$ & $b_{p}$ & $\Gamma_{p}$ & $r_{O, \kappa}$ & $r_{I, \alpha}$ & $M$ & $b_{p}$ & $\Gamma_{p}$ \\
\hline \hline 1 & $1 / 4$ & 4 & 0.50 & 1.19 & 1 & $1 / 4$ & 4 & 0.50 & 1.19 \\
& & & & & $3 / 4$ & $1 / 2$ & 4 & 0.75 & 1.39 \\
\hline 1 & $1 / 2$ & 4 & 1.00 & 1.33 & 1 & $1 / 2$ & 4 & 1.00 & 1.33 \\
& & & & & $5 / 6$ & $3 / 4$ & 4 & 1.25 & 1.52 \\
\hline 1 & $3 / 4$ & 4 & 1.50 & 1.61 & 1 & $3 / 4$ & 4 & 1.50 & 1.61 \\
& & & & & $7 / 8$ & $1 / 2$ & 16 & 1.75 & 1.51 \\
1 & $1 / 2$ & 16 & 2.00 & 1.40 & 1 & $1 / 2$ & 16 & 2.00 & 1.40 \\
\hline & & & & & $3 / 4$ & $1 / 2$ & 64 & 2.25 & 1.68 \\
& & & & & $5 / 6$ & $3 / 4$ & 16 & 2.50 & 1.76 \\
\hline \multirow{3}{*}{1} & \multirow{2}{*}{$3 / 4$} & \multirow{2}{*}{16} & 3.00 & 1.52 & 1 & $3 / 4$ & 16 & 3.00 & 1.52 \\
\hline & & & & & $5 / 6$ & $3 / 4$ & 64 & 3.75 & 1.86 \\
& & & & & 1 & $2 / 3$ & 64 & 4.00 & 1.73 \\
\hline & & & & & $4 / 5$ & $2 / 3$ & 256 & 4.27 & 1.87 \\
& & & & & 1 & $3 / 4$ & 64 & 4.50 & 1.74 \\
\hline \hline
\end{tabular}

TABLE II

THE DATA RATES REALIZED B Y TURBO-CPP QAM SCHEMES AND HSDPA MCSS

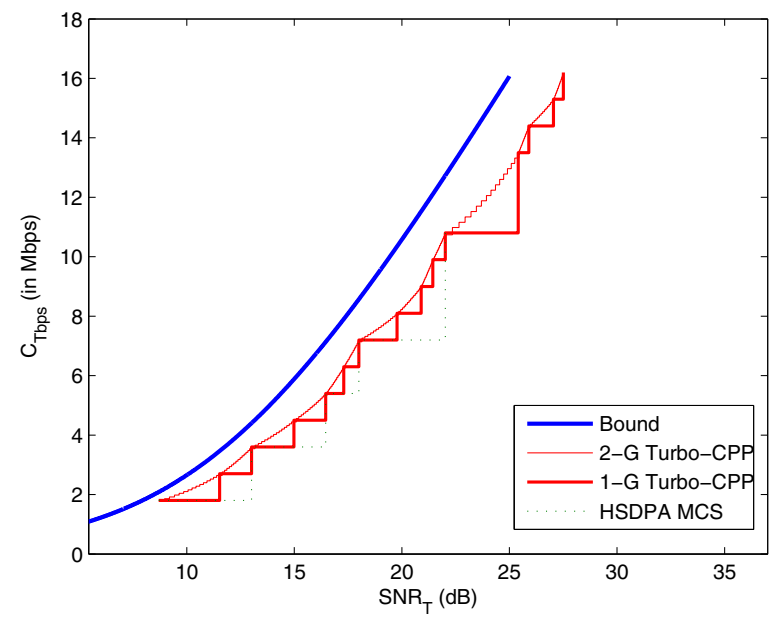

Fig. 4. The sum capacity for the HSDPA MCSs and Turbo-CPP QAM schemes with One-Group and Two-Group resource allocation schemes $(K$ $=15)$

posed MCS design results in higher sum capacities (denoted as $1-G$ Turbo-CPP) as compared to those of the HSDPA MCSs. When the two-group resource allocation scheme is in place, the sum capacities produced (denoted as 2-G Turbo-CPP) are further improved as they draw closer to the Shannon's capacity bound, which is plotted as the upper blue curve. A gain as large as $4 \mathrm{~dB}$ is achieved by the two-group resource allocation scheme implemented on the proposed MCS design over that of the HSDPA systems.

\section{CONCLUSION}

In this paper, the upper bound on the residual energy is brought down to a single-channel incremental energy as opposed to the incremental energy multiplied with the number of multi-code channels, $K$ using a two-group resource allocation scheme. As a result, the residual energy is reduced since it has been utilized to increase the sum capacity. This is further improved by decreasing the bit granularity by the rate multiplication of Turbo and CPP codes, incorporated with 4, 16, 64 and 256 QAM schemes to reduce the incremental energy, hence utilizing more residual energy to increase the sum capacity.

\section{REFERENCES}

[1] ITU. Telecommunications indicators update - 2004. [Online]. Available: http://www.itu.int/ITU-D/ict/statistics/.

[2] S. Lee, H. Lee, and D. Sung, "Capacities of single-code and multicode DS-CDMA systems accommodating multiclass services," IEEE Trans. Veh. Technol, vol. 48, no. 2, pp. 376-384, 1999.

[3] G. Forney Jr and G. Ungerboeck, "Modulation and coding for linear Gaussian channels," Information Theory, IEEE Transactions on, vol. 44, no. 6, pp. 2384-2415, 1998.

[4] J. Yang, A. Khandani, and N. Tin, "Statistical Decision Making in Adaptive Modulation and Coding for 3G Wireless Systems," Vehicular Technology, IEEE Transactions on, vol. 54, no. 6, pp. 2066-2073, 2005.

[5] S. Abedi, "Efficient radio resource management for wireless multimedia communications: a multidimensional QoS-based packet scheduler," Wireless Communications, IEEE Transactions on, vol. 4, no. 6, pp. 2811-2822, 2005

[6] J. Rhee, J. Holtzman, and D. Kim, "Performance analysis of the adaptive EXP/PF channel scheduler in an AMC/TDM system," Communications Letters, IEEE, vol. 8, no. 8, pp. 497-499, 2004.

[7] G. Aniba and S. Aissa, "Resource allocation in HSDPA using best-users selection under code constraints," in Vehicular Technology Conference, 2005. VTC 2005-Spring. 2005 IEEE 61st, vol. 1, 2005.

[8] F. Kelly, "Charging and rate control for elastic traffic: Focus on elastic services over ATM networks," European transactions on telecommunications, vol. 8, no. 1, pp. 33-37, 1997.

[9] R. Kwan, P. Chong, and M. Rinne, "Analysis of the adaptive modulation and coding algorithm with the multicode transmission," vol. 4, 2002, pp. 2007-2011 vol.4.

[10] R. Kwan and C. Leung, "Downlink scheduling optimization in CDMA networks," Communications Letters, IEEE, vol. 8, no. 10, pp. 611-613, 2004.

[11] — "Downlink Scheduling Schemes for CDMA Networks with Adaptive Modulation and Coding and Multicodes," Wireless Communications, IEEE Transactions on, vol. 6, no. 10, pp. 3668-3677, 2007.

[12] J. Zhou and M. Gurcan, "An improved multicode cdma transmission method for ad hoc networks," in Wireless Communications and Networking Conference, 2009. WCNC 2009. IEEE, April 2009, pp. 1-6.

[13] Z. He and M. Gurcan, "The rate adaptive throughput maximization in pam-modulated overloaded system," in Wireless Communications and Networking Conference, 2009. WCNC 2009. IEEE, April 2009, pp. 1-6.

[14] — "Optimizing radio resource allocation in hsdpa using 2 group allocation," in The 5th International Wireless Communications and Mobile Computing Conference, Leipzig, Germany, April 2009.

[15] 3rd Generation Partnership Project, Physical Layer Aspects of UTRA High Speed Downlink Packet Access, 3G TR25.858, 2002, [Online]. Available: http://www.3gpp.org.

[16] H. Holma, A. Toskala, T. Kolding, P. Mogensen, K. Pedersen, and J. Reunanen, WCDMA for UMTS - HSPA Evolution and LTE, 4th ed., H. Holma and A. Toskala, Eds. John Wiley \& Sons Ltd., April 2008.

[17] S. Le Goff, A. Glavieux, and C. Berrou, "Turbo-codes and high spectral efficiency modulation," 'IEEE International Conference on Communications, 1994. ICC'94, SUPERCOMM/ICC'94, Conference Record,'Serving Humanity Through Communications, vol. 2, pp. 645649, 1994.

[18] M. A. Imran and M. K. Gurcan, "Message passing algorithm for iterative decoding of channel codes," HERMIS-mu-pi International Journal of Computer Mathematics and its Applications, vol. 8, pp. 1-13, 2006.

[19] M. Luby, "Lt codes," in Foundations of Computer Science, 2002. Proceedings. The 43rd Annual IEEE Symposium on, 16-19 Nov. 2002, pp. 271-280.

[20] H. V. Poor, Wireless Communication Systems: Advanced Techniques for Signal Reception, X. Wang, Ed. Prentice Hall, 2003.

[21] H. Jenkac, J. Hagenauer, and T. Mayer, "The Turbo-Fountain," European Transactions On Telecommunications, vol. 17, no. 3, pp. 337-349, 2006. 